\title{
Effects of varying duty cycle and pulse width on high-intensity focused ultrasound (HIFU)-induced transcranial thrombolysis
}

\author{
Thilo Hölscher ${ }^{1,4^{*}}$, Rema Raman², David J Fisher ${ }^{1}$, Golnaz Ahadi ${ }^{1}$, Eyal Zadicario ${ }^{3}$ and Arne Voie ${ }^{1}$
}

\begin{abstract}
The goal was to test the effects of various combinations of pulse widths (PW) and duty cycles (DC) on high-intensity focused ultrasound (HIFU)-induced sonothrombolysis efficacy using an in vitro flow model. An ExAblate $^{\text {TM }} 4000$ HIFU headsystem (InSightec, Inc., Israel) was used. Artificial blood clots were placed into test tubes inside a human calvarium and exposed to pulsatile flow. Four different duty cycles were tested against four different pulse widths. For all study groups, an increase in thrombolysis efficacy could be seen in association with increasing DC and/or PW $(p<0.0001)$. Using transcranial HIFU, significant thrombolysis can be achieved within seconds and without the use of lytic drugs in vitro. Longer duty cycles in combination with longer pulse widths seem to have the highest potential to optimize clot lysis efficacy.
\end{abstract}

Keywords: Transcranial, High-intensity focused ultrasound, Thrombolysis, Stroke, Pulse width, Duty cycle

\section{Background}

Reversible disaggregation of fibrin fibers, improved distribution of plasminogen, tissue plasminogen activator (tPA) within a blood clot, and cavitational mechanisms have been described to explain why ultrasound enhances clot lysis [1-5]. Early clinical studies on transcranial sonothrombolysis in stroke patients using diagnostic ultrasound devices were promising. The most relevant work, so far, was published in 2004 by Alexandrov et al. [6]. Other studies showed comparable results [7-12], suggesting that the use of transcranial diagnostic ultrasound in combination with TPA shortens the time to recanalize vessels significantly. Despite the excitement for this new treatment approach, it is limited to a minority of patients since less than $3 \%$ of all stroke victims currently receive tPA. The successful use of HIFU for sonothrombolysis outside the cranium and without the additional use of lytic agents, such as tPA, has been described earlier [13]. Very recent data showing the feasibility of HIFU for transcranial sonothrombolysis in the

\footnotetext{
* Correspondence: thoelscher@ucsd.edu

${ }^{1}$ Brain Ultrasound Research Laboratory (BURL), Department of Radiology,

University of California San Diego, San Diego, CA, 92103, USA

${ }^{4}$ Department of Neurosciences, University of California San Diego, 200 West

Arbor Drive, San Diego, CA, 92103-8756, USA

Full list of author information is available at the end of the article
}

absence of tPA [14] demonstrate its future potential as a noninvasive, drug-independent treatment option for stroke. However, the data describing the transcranial application for potential use in stroke patients are sparse. Regarding the various ultrasound operating parameters, such as transmit frequency, acoustic output power, duty cycle, and pulse width, it is unclear which combination of these parameters might be preferable to gain efficacious sonothrombolysis in a safe manner, trying to avoid uncontrolled thermal as well as cavitational effects which are most feared in this regard. Hence, thorough research has to be done to define the most efficacious combinations of ultrasound operating parameters to be applied in such a fashion that thermal as well as cavitation effects can be either avoided or, at least, controlled. To date, the knowledge about sonothrombolysis parameter optimization is very limited. One of the most relevant works in this regard was published by Schafer et al. [15]. They tested the effect of operating parameters on the thrombolytic potency of ultrasound and found that thrombolytic efficiency of ultrasound depends directly on duration, intensity, duty cycle, and pulse length, and inversely on frequency. It is important to mention, however, that the knowledge gained by Schafer et al. is based on transmit frequencies between 2.0 and $4.5 \mathrm{MHz}$. The 
transmit frequency of the device used for the present study is $220 \mathrm{kHz}$. Therefore, it is questionable whether the results can be translated to low-frequency ultrasound systems. The goal of this study was to investigate the impact of varying duty cycles and pulse width on HIFU-induced thrombolysis efficacy, in the absence of tPA, in a transcranial in vitro flow model.

\section{Methods}

Ultrasound system and operating parameters

The ExAblate $^{\mathrm{TM}} 4000$ (InSightec Inc., Tirat Carmel, Israel) HIFU headsystem has been developed for brain applications. A key component of this system is a hemispheric phased array transducer with 1,000 single piezo elements that can be operated independently. Due to its geometry, a sharp focus can be generated, located in the center of the transducer (Figure 1). The transmit frequency of the system is $220 \mathrm{kHz}$.

To test the impact of various duty cycles and pulse widths on thrombolysis, the decision was made to retain an insonation duration of $30 \mathrm{~s}$ and an acoustic output power of $235 \mathrm{~W}$ for all studies. The parameters above were chosen due to own data in [14], which show that transcranial thrombolysis can be achieved using these parameters. In the present study, four different duty cycles were tested in combination with four different pulse widths, as displayed in Table 1.

\section{Clot preparation}

Venous whole blood was drawn from healthy, unmedicated donors and transferred into citrate tubes. The donors were enrolled after signing a written informed consent according to the local Institutional Review Board approval (Internal Review Board of the University of California, San Diego). For each clot, $0.5 \mathrm{ml}$ citrate blood was mixed with $40 \mu \mathrm{CaCl}_{2}(210 \mathrm{mmol} / \mathrm{l})$ and transferred into a borosilicate glass tube, which had a silk thread inside. The clots were incubated for $3 \mathrm{~h}$ in a $37^{\circ} \mathrm{C}$ water bath. The thrombus was organized around the silk thread so that it will be kept in place inside the test tube during insonation. The thrombi had an average weight of $0.2519 \mathrm{~g} \pm 7 \%$. The average length of each thrombus was about $2.5 \mathrm{~cm}$.

\section{Experimental setup}

A degassed, cadaveric skull specimen was mounted on an acrylic frame and placed upside down into the degassed water-filled cavity of the hemispheric transducer. The thrombus inside the test tube was placed at the natural focus of the transducer, which is located at the geometrical center in $X / Y$ and $15 \mathrm{~cm}$ from the bottom in the $Z$ orientation of the hemispheric array (Figure 2). For all experiments, the clots were exposed to circulating, pulsatile flow $(10 \mathrm{ml} / \mathrm{min})$. Phosphate buffered

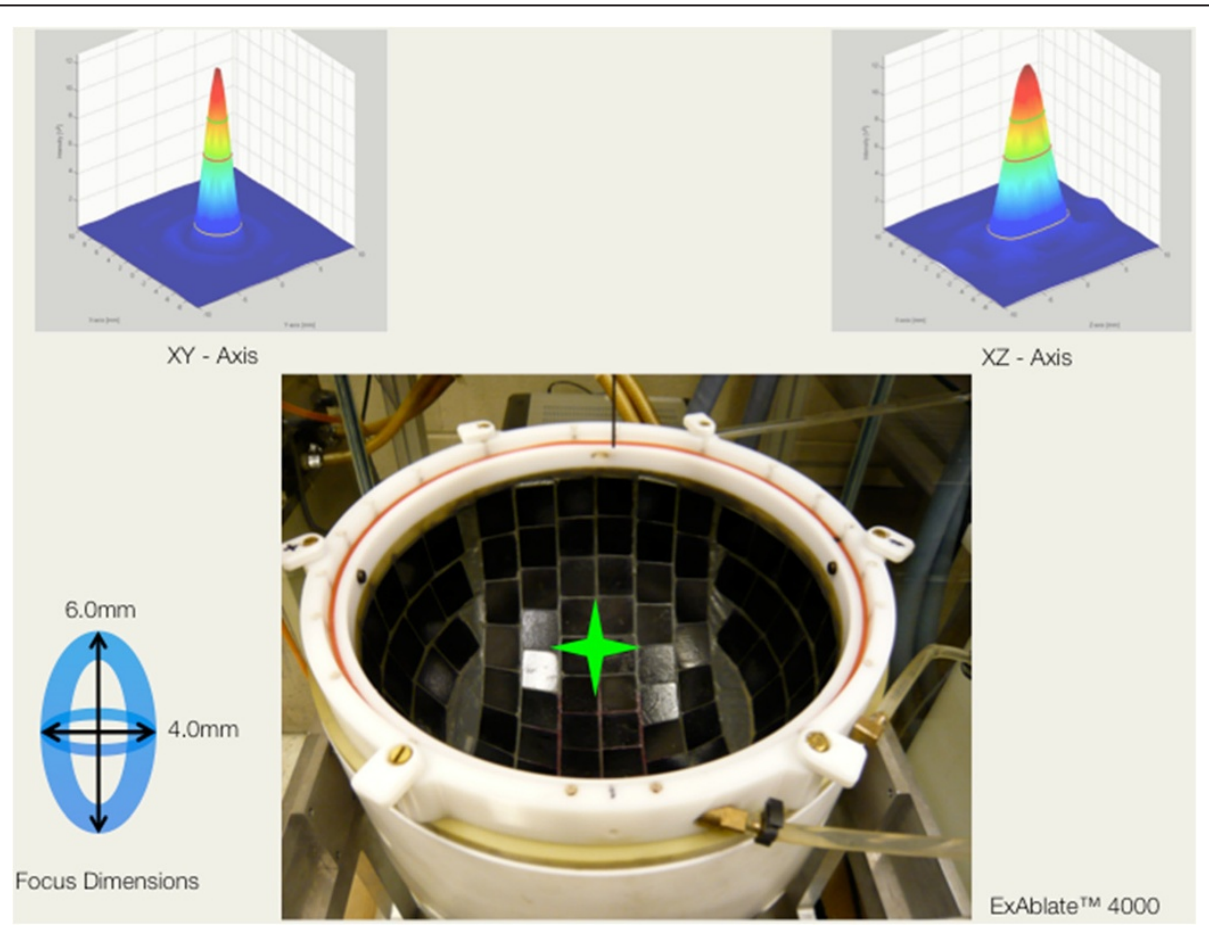

Figure 1 Top view inside the hemispheric transducer of the ExAblate ${ }^{\mathrm{TM}} \mathbf{4 0 0 0}$ HIFU headsystem. Each black tile contains nine single ultrasound-transmitting elements (total $N=1,000$ ). All elements transmit towards the center of the transducer (green star), creating a sharp focus beam of 4.0-mm diameter in lateral and $6.0 \mathrm{~mm}$ in elevational orientation. Parametric images of the focus, based on acoustic measurements, are given in $X Y$ (upper left) and $Z$ (upper right) orientations. 
Table 1 Combinations of DC, PW, and the total $N$ per study group

\begin{tabular}{ccccc}
\hline DC (\%) & \multicolumn{4}{c}{ PW } \\
\cline { 2 - 5 } & $\mathbf{0 . 1} \mathbf{~ m s}$ & $\mathbf{1 ~} \mathbf{~ s}$ & $\mathbf{1 0} \mathbf{~} \mathbf{s}$ & $\mathbf{1 0 0} \mathbf{~ m s}$ \\
\hline 5 & $N=40$ & $N=40$ & $N=40$ & $N=40$ \\
10 & $N=40$ & $N=44$ & $N=40$ & $N=40$ \\
20 & $N=41$ & $N=40$ & $N=41$ & $N=43$ \\
50 & $N=43$ & $N=40$ & $N=42$ & $N=44$ \\
\hline
\end{tabular}

$D C$, duty cycles; $P W$, pulse width.

saline was used as a fluid medium. The ambient fluid temperature inside the hemispheric transducer as well as inside the test tube was kept at $24^{\circ} \mathrm{C}$.

\section{Acoustic field mapping}

For each of the 16 different duty cycle (DC)/pulse width (PW) combinations, the acoustic parameters spatial peak temporal average intensity $\left(I_{\text {SPTA }}\right)$, peak negative pressure $\left(P_{\mathrm{NEG}}\right)$, and peak positive pressure $\left(P_{\mathrm{POS}}\right)$ were measured using a HIFU hydrophone (Model Y120, Sonic Concepts, Seattle, WA), calibrated for the frequency of $220 \mathrm{kHz}$. The acoustic measurements are presented in Table 2.

\section{Statistical analysis}

Percent weight loss within each of the 16 DC/PW combinations was compared using a Wilcoxon signed-rank test. A Kruskal-Wallis test was used to examine the differences in clot weight loss among the four PW settings within each DC group. If the overall difference was statistically significant, pair-wise comparisons were performed using a Wilcoxon rank-sum test with the $p$ values adjusted using a Bonferroni-Holm correction for multiple comparisons.

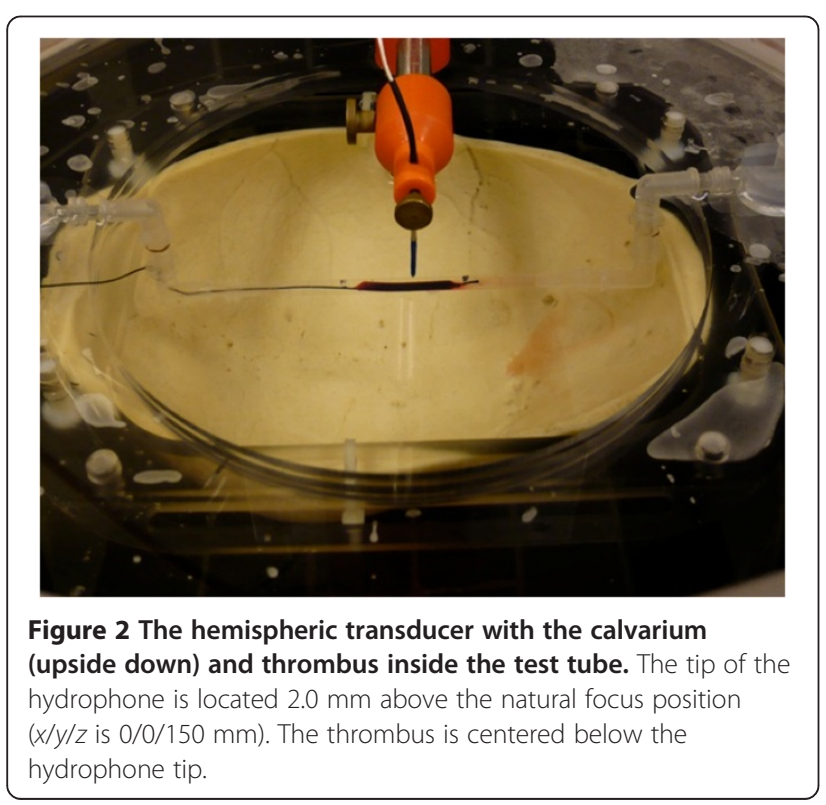

Table $2 I_{\text {SPTA, }} P_{\text {NEG }}$ and $P_{\text {POs }}$ pressure values for all 16 parameter combinations

\begin{tabular}{ccccc}
\hline $\begin{array}{c}\text { Study } \\
\text { group }\end{array}$ & $\begin{array}{c}\boldsymbol{I}_{\text {SPTA }} \\
\left(\mathbf{W} / \mathbf{c m}^{2}\right)\end{array}$ & $\begin{array}{c}\boldsymbol{P}_{\text {NEG }} \\
(\mathbf{M P a})\end{array}$ & $\begin{array}{c}\boldsymbol{P}_{\text {POS }} \\
(\mathbf{M P a})\end{array}$ & $\begin{array}{c}\boldsymbol{N} \text { per } \\
\text { group }\end{array}$ \\
\hline 1 & 13.7 & 2.8 & 3.1 & 40 \\
2 & 13.7 & 2.8 & 3.1 & 40 \\
3 & 13.7 & 2.8 & 3.1 & 43 \\
4 & 13.7 & 2.8 & 3.1 & 43 \\
5 & 27.4 & 2.8 & 3.1 & 43 \\
6 & 27.4 & 2.8 & 3.1 & 43 \\
7 & 27.4 & 2.8 & 3.1 & 43 \\
8 & 27.4 & 2.8 & 3.1 & 43 \\
9 & 54.7 & 2.8 & 3.1 & 43 \\
10 & 54.7 & 2.8 & 3.1 & 43 \\
11 & 54.7 & 2.8 & 3.1 & 43 \\
12 & 54.7 & 2.8 & 3.1 & 43 \\
13 & 136.8 & 2.8 & 3.1 & 43 \\
14 & 136.8 & 2.8 & 3.1 & 43 \\
15 & 136.8 & 2.8 & 3.1 & 43 \\
16 & 136.8 & 2.8 & 3.1 & 44 \\
\hline
\end{tabular}

\section{Results}

Overall, a total of $N=658$ experiments were performed. The results are given in Table 3 . For each study group, the values for average percent weight loss, standard deviation, and percent weight loss per kilo Joule are given. Three main observations could be made: (1) For all study groups, the percent weight loss was statistically significant $(p<0.001)$. (2) With increasing DCs as well as increasing PWs, the percent weight loss increased as well. (3) At a DC of 50\%, the change of PW did not lead to a significant change in weight loss.

A visual presentation of the percent weight loss for each group, displayed as boxplots, is given in Figure 3.

\section{Discussion}

Disregarding the acoustic output power or insonation duration, it could be demonstrated in vitro that sonothrombolysis efficacy using transcranial high-intensity focused ultrasound is dependent on the combination of duty cycle and pulse width. Looking at clot weight loss over time, increased duty cycles in combination with increased pulse widths seem to be preferable.

The observation of higher clot weight loss with increased duty cycles and pulse widths might be rather obvious at first sight. As shown in Table 2, the $I_{\text {SPTA }}$ increases with higher duty cycles, whereas the $I_{\text {SPTA }}$ for a $5 \%$ duty cycle is $13.7 \mathrm{~W} / \mathrm{cm}^{2}$; in combination with any given pulse width, it increases to tenfold using a duty cycle of $50 \%\left(136.8 \mathrm{~W} / \mathrm{cm}^{2}\right)$. Accordingly, the energy deposition at the clot site increases from 100 to $1,000 \mathrm{~J}$. 
Table $3 \%$ WT loss mean, Std dev, and pulse 'off time' are given for all 16 parameter combinations

\begin{tabular}{|c|c|c|c|c|c|}
\hline & DC (\%) & & & & \\
\hline & & $0.1 \mathrm{~ms}$ & $1 \mathrm{~ms}$ & $10 \mathrm{~ms}$ & $100 \mathrm{~ms}$ \\
\hline & 5 & GP 1 & GP 2 & GP 3 & GP 4 \\
\hline$N$ & & 40 & 40 & 40 & 40 \\
\hline \% Wt loss (mean) & & 10.28 & 17.55 & 23.62 & 27.15 \\
\hline Std dev & & 11.95 & 7.08 & 11.97 & 20.75 \\
\hline Off time (ms) & & 1.9 & 19 & 190 & 1900 \\
\hline & 10 & GP 5 & GP 6 & GP 7 & GP 8 \\
\hline$N$ & & 40 & 44 & 40 & 40 \\
\hline \% Wt loss (mean) & & 17.12 & 24.32 & 29.08 & 42.92 \\
\hline Std dev & & 9.1 & 15.88 & 17.33 & 24.67 \\
\hline Off time (ms) & & 0.9 & 9 & 90 & 900 \\
\hline & 20 & GP 9 & GP 10 & GP 11 & GP 12 \\
\hline$N$ & & 41 & 40 & 41 & 43 \\
\hline \% Wt loss (mean) & & 30.02 & 37.7 & 39.9 & 59.6 \\
\hline Std dev & & 20.12 & 21.83 & 21.5 & 19.16 \\
\hline Off time (ms) & & 0.4 & 4 & 40 & 400 \\
\hline & 50 & GP 13 & GP 14 & GP 15 & GP 16 \\
\hline$N$ & & 43 & 40 & 42 & 44 \\
\hline$\%$ Wt loss (mean) & & 48.67 & 53.58 & 51.43 & 59.23 \\
\hline Std dev & & 23.7 & 20.85 & 21.85 & 17.1 \\
\hline Off time (ms) & & 0.2 & 2 & 20 & 200 \\
\hline
\end{tabular}

The italicized entries represent the 16 different study groups, i.e., GP14 is study group \#14.\% WT loss, percent weight loss; Std dev, standard deviation.
This observation is in accordance, to some extent, with the work published by Schafer et al. [15]. Using a nonfocused, diagnostic ultrasound transducer in an in vitro sonothrombolysis setup, three different duty cycles were tested in combination with increasing pulse widths between 100 and $400 \mu \mathrm{s}$. With regard to the parameter duty cycle, the highest amount of thrombolysis efficacy was seen using a $100 \%$ duty cycle, i.e., continuous wave mode. The smallest amount of clot lysis was seen using a $20 \%$ duty cycle. With regard to the operating parameter pulse width, the investigators described only a modest effect. Given a constant duty cycle, increased pulse widths caused higher clot weight loss as well, however, to less extent. This could be confirmed in the present study. A possible reason why an increase in clot weight loss can be observed with greater pulse widths, although duty cycle, intensity, and total energy remain constant, might be that the total number of pulses per insonation duration decreases. That implies that each pulse simply contains more energy and, therefore, presumably improves clot/fibrin disaggregation. Noteworthy is the observation that increased clot weight loss was rather distinct using short duty cycles in combination with increased pulse widths, whereas the change of pulse width using longer duty cycles, such as $50 \%$ in the present study, diminished.

In the present study, only the parameters duty cycle and pulse length were changed, whereas the parameters acoustic output power and insonation duration remained unchanged. Accordingly, the acoustic intensities varied between the study groups with the lowest $I_{\mathrm{SPTA}}$ seen in the $5 \% \mathrm{DC}$ and the highest $I_{\mathrm{SPTA}}$ values in the $50 \%$ duty cycle groups, as shown in Table 2. Rosenschein et al. [13] followed a similar approach to investigate the potential

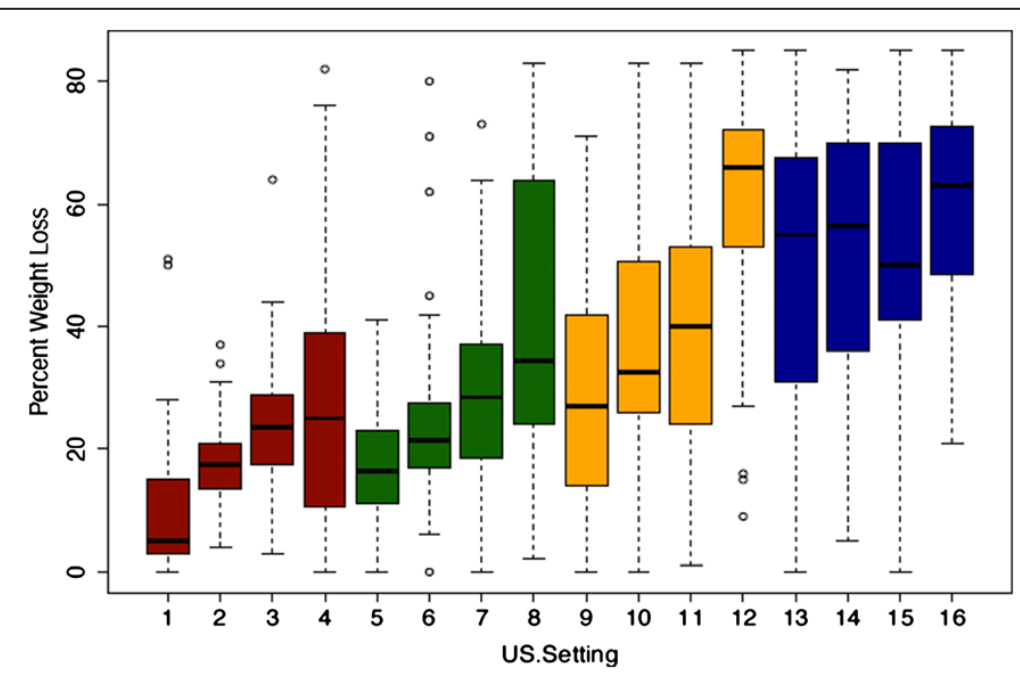

Figure 3 Histogram showing the percent clot weight loss for all 16 study groups. 5\% DC (red bars), 10\% DC (green bars), 20\% DC (yellow bars), and 50\% DC (blue bars). 
impact of duty cycle and pulse length on clot lysis efficacy. They tested five pulse lengths $(50,100,200,300$, and $400 \mu \mathrm{s})$ against various duty cycles $(10 \%, 5 \%, 4 \%, 3.3 \%$, and 2.5\%), but-different from the present study-they adapted the acoustic output power to keep the $I_{\text {SPTA }}$ at the focal spot constant at $40 \mathrm{~W} / \mathrm{cm}^{2}$ throughout all study groups. The total insonation duration was $4 \mathrm{~min}$ and remained unchanged as well. Differing from the present study, they found an optimized clot lysis efficiency using a short duty cycle of $4 \%$ and a pulse length of $200 \mu \mathrm{s}$.

The major limitation of the present study is the missing translation into an appropriate in vivo model. A sonothrombolysis rabbit model has been established recently to investigate whether the rather promising in vitro results can be confirmed in a living system. Of greatest importance will be to investigate whether this application might cause vascular damage or unwanted blood-brain barrier opening, and if the latter should be the case, what could be done to significantly improve the safety profile. In addition, temperature assessments are missing in the present work. Although there is a common belief that the underlying mechanism of clot/ fibrin disaggregation is of mechanical nature, thermal effects cannot be excluded until appropriate measurements or appropriate calculations are available, more so since temperature elevation during sonothrombolysis would be a serious safety issue. Regarding safety, potential clot fragmentation is of concern as well.

\section{Conclusion}

Using transcranial HIFU, significant thrombolysis can be achieved within seconds and without the use of lytic drugs. Aiming ultrasound operating parameter optimization, the present data show that the combination of long duty cycles and long pulse widths seems preferable. A duty cycle of $50 \%$ seems to represent an upper limit above which a change of the pulse width does not affect the thrombolysis efficacy. The current findings might be taken into consideration for future translational research on HIFU-induced thrombolysis. The development of a noninvasive, transcranial ultrasound technology which provides the possibility to treat stroke victims who are not eligible for tPA would be a significant achievement in today's stroke care.

\section{Competing interests}

The authors declare that they have no competing interests.

\section{Authors' contributions}

TH designed the study and the experimental setup, supervised all aspects of the project, and wrote the manuscript. RR did the statistical work-up of the data. DJF was involved in the experimental setup and supervised and participated in the data collection. GA acquired most of the data and prepared them for statistical analysis. EZ participated in the planning of the experiment and provided technical support. AV performed all acoustic measurements and contributed to the experimental design and manuscript revisions. All authors read and approved the final manuscript.

\section{Acknowledgements}

This work was supported by InSightec, Inc., Tirat Carmel, Israel, and by the National Institute of Health (grant number R01HL091043).

\section{Author details}

'Brain Ultrasound Research Laboratory (BURL), Department of Radiology, University of California San Diego, San Diego, CA, 92103, USA. ${ }^{2}$ Division of Biostatistics and Bioinformatics, University of California San Diego, San Diego, CA, 92093, USA. ${ }^{3}$ InSightec, Ltd, 5 Nahum Heth St., Tirat Carmel, 39120, Israel. ${ }^{4}$ Department of Neurosciences, University of California San Diego, 200 West Arbor Drive, San Diego, CA, 92103-8756, USA.

Received: 22 April 2013 Accepted: 4 August 2013

Published: 1 October 2013

\section{References}

1. Braaten JV, Goss RA, Francis CW. Ultrasound reversibly disaggregates fibrin fibers. Thromb Haemost. 1997; 78(3):1063-8.

2. Devcic-Kuhar B, Pfaffenberger S, Gherardini L, Mayer C, Groschl M, Kaun C, Benes E, Tschachler E, Huber K, Maurer G, Wojta J, Gottsauner-Wolf M. Ultrasound affects distribution of plasminogen and tissue-type plasminogen activator in whole blood clots in vitro. Thromb Haemost. 2004; 92(5):980-5.

3. Datta S, Ammi AY, Coussios CC, Holland CK. Monitoring and simulating stable cavitation during ultrasound-enhanced thrombolysis. J Acoust Soc Am. 2007; 122:3052.

4. Datta S, Coussios CC, McAdory LE, Tan J, Porter T, De Courten-Myers G, Holland CK. Correlation of cavitation with ultrasound enhancement of thrombolysis. Ultrasound Med Biol. 2006; 32(8):1257-67.

5. Prokop AF, Soltani A, Roy RA. Cavitational mechanisms in ultrasoundaccelerated fibrinolysis. Ultrasound Med Biol. 2007; 33(6):924-33.

6. Alexandrov AV, Molina CA, Grotta JC, Garami Z, Ford SR, Alvarez-Sabin J, Montaner J, Saqqur M, Demchuk AM, Moyé LA, Hill MD, Wojner AW, CLOTBUST Investigators. Ultrasound-enhanced systemic thrombolysis for acute ischemic stroke. N Engl J Med. 2004; 351(21):2170-8.

7. Alexandrov AV, Mikulik R, Ribo M, Sharma VK, Lao AY, Tsivgoulis G, Sugg RM, Barreto A, Sierzenski P, Malkoff MD, Grotta JC. A pilot randomized clinical safety study of sonothrombolysis augmentation with ultrasound-activated perflutrenlipid microspheres for acute ischemic stroke. Stroke. 2008; 39(5):1464-9.

8. Eggers J, Seidel G, Koch B, Konig IR. Sonothrombolysis in acute ischemic stroke for patients ineligible for rt-PA. Neurology. 2005; 64(6):1052-4.

9. Eggers J, Konig IR, Koch B, Handler G, Seidel G. Sonothrombolysis with transcranial color-coded sonography and recombinant tissue-type plasminogen activator in acute middle cerebral artery main stem occlusion: results from a randomized study. Stroke. 2008; 39(5):1470-5.

10. Dinia L, Rubiera M, Ribo M, Maisterra O, Ortega G, del Sette M, Alvarez-Sabin J, Molina CA. Reperfusion after stroke sonothrombolysis with microbubbles may predict intracranial bleeding. Neurology. 2009; 73(10):775-80.

11. Molina CA, Barreto AD, Tsivgoulis G, Sierzenski P, Malkoff MD, Rubiera M, Gonzales N, Mikulik R, Pate G, Ostrem J, Singleton W, Manvelian G, Unger EC, Grotta JC, Schellinger PD, Alexandrov AV. Transcranial ultrasound in clinical sonothrombolysis (TUCSON) trial. Ann Neurol. 2009; 66(1):28-38

12. Molina CA, Ribo M, Rubiera M, Montaner J, Santamarina E, DelgadoMederos R, Arenillas JF, Huertas R, Purroy F, Delgado P, Alvarez-Sabín J. Microbubble administration accelerates clot lysis during continuous 2-MHz ultrasound monitoring in stroke patients treated with intravenous tissue plasminogen activator. Stroke. 2006; 37(2):425-9.

13. Rosenschein U, Furman V, Kerner E, Fabian I, Bernheim J, Eshel Y. Ultrasound imaging-guided noninvasive ultrasound thrombolysis: preclinical results. Circulation. 2000; 102(2):238-45.

14. Holscher T, Fisher D, Raman R, Ernstrom K, Zadicario E, Bradley W, Voie A. Noninvasive transcranial clot lysis using high intensity focused ultrasound. J Neurol Neurophysiol. 2011. doi:10.4172/2155-9562.S1-002.

15. Schafer S, Kliner S, Klinghammer L, Kaarmann H, Lucic I, Nixdorff U, Rosenschein U, Daniel WG, Flachskampf FA. Influence of ultrasound operating parameters on ultrasound-induced thrombolysis in vitro. Ultrasound Med Biol. 2005; 31(6):841-7.

doi:10.1186/2050-5736-1-18

Cite this article as: Hölscher et al:: Effects of varying duty cycle and pulse width on high-intensity focused ultrasound (HIFU)-induced transcranial thrombolysis. Journal of Therapeutic Ultrasound 2013 1:18. 